\title{
Second-harmonic generation from complementary split-ring resonators
}

\author{
N. Feth, ${ }^{1,2, *}$ S. Linden, ${ }^{1,2,3}$ M. W. Klein, ${ }^{2,3}$ M. Decker, ${ }_{4}^{2,3}$ F. B. P. Niesler, ${ }_{1,2}^{3}$ Y. Zeng, ${ }^{4}$ W. Hoyer, ${ }^{5}$ J. Liu, $^{4}$ \\ S. W. Koch, ${ }^{5}$ J. V. Moloney, ${ }^{4}$ and M. Wegener ${ }^{1,2,3}$ \\ ${ }^{1}$ Institut für Nanotechnologie, Forschungszentrum Karlsruhe in der Helmholtz-Gemeinschaft, \\ D-76021 Karlsruhe, Germany \\ ${ }^{2}$ DFG-Center for Functional Nanostructures, Universität Karlsruhe (TH), D-76128 Karlsruhe, Germany \\ ${ }^{3}$ Institut für Angewandte Physik, Universität Karlsruhe (TH), D-76128 Karlsruhe, Germany \\ ${ }^{4}$ Arizona Center for Mathematical Sciences, University of Arizona, Tucson, Arizona 85721, USA \\ ${ }^{5}$ Department of Physics and Material Sciences Center, Philipps University, Renthof 5, D-35032 Marburg, Germany \\ *Corresponding author: Nils.Feth@physik.uni-karlsruhe.de
}

Received June 20, 2008; revised July 25, 2008; accepted July 29, 2008; posted August 5, 2008 (Doc. ID 97741); published August 25, 2008

We present experiments on second-harmonic generation from arrays of magnetic split-ring resonators and arrays of complementary split-ring resonators. In both cases, the fundamental resonance is excited by the incident femtosecond laser pulses under normal incidence, leading to comparably strong second-harmonic signals. These findings are discussed in terms of Babinet's principle and in terms of a recently developed microscopic classical theory that leads to good agreement regarding the relative and the absolute nonlinear signal strengths. The hydrodynamic convective contribution is found to be the dominant source of secondharmonic generation-in contrast to a previous assignment [Science 313, 502 (2006)]. ㅇ 2008 Optical Society of America

OCIS codes: $160.4330,190.3970,260.5740$.

Photonic metamaterials are an emerging class of tailored composite effective materials that can provide interesting optical properties such as, e.g., magnetism at elevated frequencies [1-3] or an enhanced nonlinear optical response [1,4-10]. Indeed, in a series of recent experiments [6-8], we have observed second-harmonic generation (SHG) from planar arrays of gold split-ring resonators (SRRs) exhibiting a pronounced magnetic-dipole response. In essence, a SRR is an almost closed loop of a metal wire that can be viewed as a subwavelength electromagnet in which the incident light field induces a circulating oscillating electrical current, leading to a local magnetic field (magnetic-dipole moment) perpendicular to the SRR plane. It has been found $[7,8]$ that arrays of gold "T"s show SHG that is 2 orders of magnitude smaller than that of the gold SRR arrays-despite the facts that the "T"s also exhibit a resonance at the incident fundamental laser frequency and that they are also known to exhibit pronounced local field enhancements.

In this Letter, we aim at further clarifying the underlying mechanism. First, we present new experimental results on complementary split-ring resonators (CSRRs), i.e., SRRs in which the metal in the sample plane is replaced by air and vice versa. According to the generalized Babinet's principle [11,12], magnetic and electric fields are interchanged with respect to SRR (precisely, $\vec{E}(\vec{r}, t) \leftrightarrow-c_{0} \vec{B}(\vec{r}, t)$ ). Hence, the magnetic-dipole moment of the SRR turns into an electric-dipole moment of the CSRR, allowing us to investigate whether the magnetic-dipole moment is crucial for efficient SHG. Second, we compare these experimental results with numerical calculations based on a recently developed microscopic classical theory of the metal-based metamaterial optical nonlinearities [13].

The samples for our experiments are fabricated by standard electron-beam lithography, electron-beam evaporation of the $25 \mathrm{~nm}$ thick gold layer onto a glass substrate coated with a $5 \mathrm{~nm}$ thin film of indium tin oxide (ITO), and subsequent lift-off. Details can be found in [14]. The SRR and the CSRR arrays have been fabricated on two different glass substrates, however, with closely similar processing steps. The footprint of each array is $100 \mu \mathrm{m} \times 100 \mu \mathrm{m}$. Electron micrographs of representative regions of the SRR sample and of the CSRR sample are shown in Fig. 1.

Figure 1 also shows the linear transmittance (solid) and reflectance (dashed) spectra measured with a commercial Fourier-transform microscope spectrometer under normal incidence of light. The black arrows indicate the incident linear polarization. As expected from Babinet's principle and in agreement with the literature [11,12], interchanging $\mathrm{SRR} \leftrightarrow \mathrm{CSRR}$ is accompanied by interchanging transmittance $\leftrightarrow$ reflectance and by interchanging horizontal $\leftrightarrow$ vertical incident linear polarization. In particular, Babinet's principle applies to the fundamental resonance at around $1.5 \mu \mathrm{m}$ wavelength.

In our SHG experiments, femtosecond pulses obtained from an optical parametric oscillator (Spectra Physics OPAL) are tuned to $1.5 \mu \mathrm{m}$ wavelength and focused onto these metamaterial arrays under normal incidence. The duration of the Gaussian pulses is $170 \mathrm{fs}$, and their repetition rate is $81 \mathrm{MHz}$. Typically, an average power of $90 \mathrm{~mW}$ impinges onto the samples (equivalent to $6.8 \times 10^{17}$ photons $/ \mathrm{s}$ at $0.826 \mathrm{eV}$ photon energy). The $1 / e^{2}$ intensity diameter of the Gaussian focal spot is measured as $60 \mu \mathrm{m}$ by a 

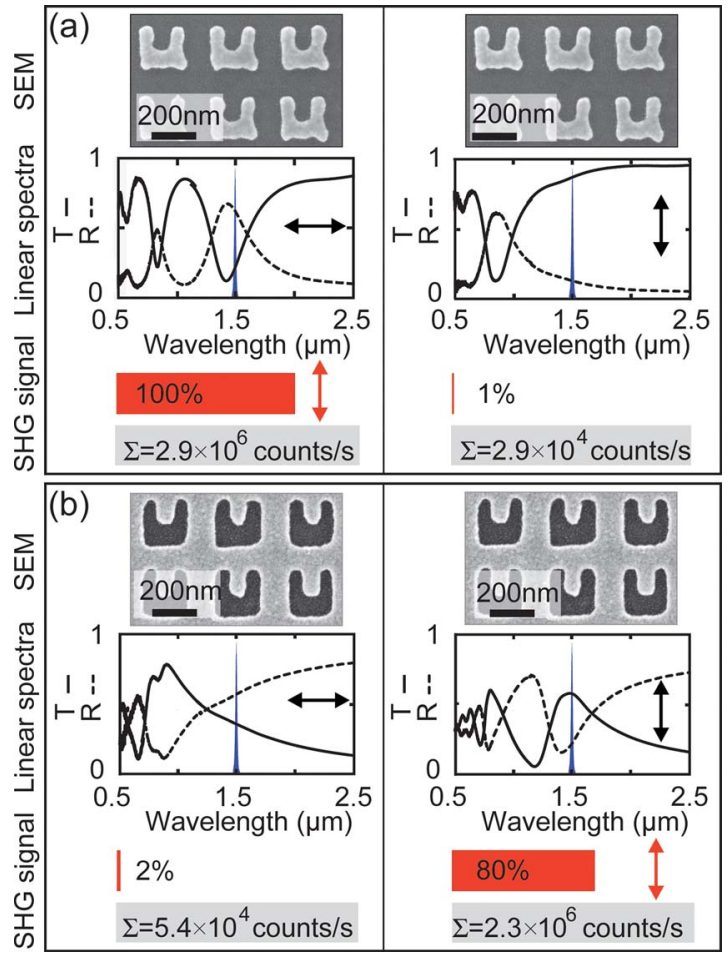

Fig. 1. (Color online) Experiments on (a) split-ring resonators and (b) complementary split-ring resonators. The different rows (from top to bottom) show scanning electron micrographs, linear transmittance $T$ (solid) and reflectance $R$ (dashed) spectra (black), and the relative measured SHG signals [bars labeled with percents (red online)]. The corresponding rates $\Sigma$ are the measured integral number of counts per second. The arrows indicate the incident linear polarization (dark), the measured linear polarization of the SHG [gray (red online)]-if sufficiently large. The shaded areas (blue online) in the linear spectra illustrate the exciting laser spectrum centered at the $1.5 \mu \mathrm{m}$ wavelength.

knife-edge technique. Under these conditions, the SHG signal scales with the square of the incident power. The incident linear polarization is controlled by a half-wave plate and a polarizer. The SHG signal is detected in the forward direction, spectrally dispersed by a $0.25 \mathrm{~m}$ grating spectrometer, and recorded via a sensitive liquid-nitrogen-cooled silicon CCD camera. Here, we do not depict the measured spectra (that merely reveal the expected Gaussian maximum centered around the second harmonic) but rather discuss the integral number of counts. A polarizer in front of the spectrometer allows for measuring the polarization of the emerging SHG signal. More details can be found in [6-8]. We estimate that, due to the CCD camera quantum efficiency, the grating efficiency, and various losses in the optical setup, the number of photons emitted from the sample is roughly a factor of 3 higher than the quoted number of counts. The central experimental results of this Letter are the SHG signals shown in Fig. 1. For example, $2.9 \times 10^{6}$ counts/s correspond to an estimated SHG power conversion efficiency of $\eta=3 \times 10^{-11}$ (also see [8]). The SHG signal from the CSRR is comparable with that of the SRR. The gray arrows (red online) indicate the measured linear polarization of the emerging SHG signal. As expected from symmetry, the SHG polarization is oriented along the vertical axis (broken inversion symmetry) for both the SRR as well as for the CSRR. These results have been reproduced several times by interchanging the SRR and the CSRR samples back and forth and by repeating the experiment. Following Babinet's principle, the CSRR reveals no magnetic-dipole moment under these conditions, yet the CSRRs lead to comparable SHG signals. This observation leads us to conclude that there is generally no correlation between SHG efficiency and the magnetic-dipole moment. This correlation does exist for all of our previous experiments $[6,7]$, yet it is wrong in general.

To further clarify the nature of the SHG signal emerging from these metallic metamaterials, we compare the experiments with a recently developed microscopic theory [13]. The nonlinear response is exclusively based on the metal classical electron gas. In order to connect to the electromagnetic fields in the Maxwell equations the velocity of individual electrons needs to be translated via a velocity field $\vec{v}(\vec{r}, t)$ into a current-density field $\vec{j}(\vec{r}, t)$. In close analogy to hydrodynamics, the total time derivative in Newton's second law leads to an additional convective contribution ("cold-plasma equations" in plasma physics [15]). It has been found that this convective contribution dominates the SHG response of SRR [13]. Here, we apply the same approach to CSRR. As usual, the complete equations are evaluated within a perturbative approach up to second order ("(2)") in the incident fields, leading to

$$
\begin{aligned}
\frac{\partial \vec{j}_{2 \omega}^{(2)}}{\partial t}= & \varepsilon_{0} \omega_{\mathrm{pl}}^{2} \vec{E}_{2 \omega}^{(2)}-\omega_{\mathrm{col}} \vec{j}_{2 \omega}^{(2)} \\
& -\frac{\varepsilon_{0} \mathrm{e}}{m}\left(\vec{\nabla} \cdot \vec{E}^{(1)}\right) \vec{E}^{(1)}-\frac{\mathrm{e}}{m} \vec{j}^{(1)} \vec{B}^{(1)}+\sum_{k=1}^{3} \frac{\partial \vec{j}^{(1)} j_{k}^{(1)}}{\partial r_{k}} \frac{\mathrm{e} n_{\mathrm{i}}}{}
\end{aligned}
$$

Here, $\omega_{\mathrm{pl}}$ is the metal plasma frequency, $\omega_{\text {coll }}$ is the electron collision frequency, $n_{\mathrm{i}}$ is the ion density, $m$ is the electron mass, and e is the elementary charge. The first two terms are linear. They describe the backaction of the SHG field on the classical electron gas and the usual collisional damping, respectively. The following terms are nonlinear. The third and fourth terms originate from the electric and magnetic component of the Lorentz force, respectively. The former stems from a light-induced change of the charge density near the metal surface, and the latter has been the basis of our previous nonlinear modeling [6]. The last term is the convective contribution that describes the change of the electron momentum induced by the variation of the local electron density due to the incident light field. Although not intuitive at first sight, in analogy to usual hydrodynamics, the convective term is a nonlinear source even without any actual nonlinear force exerted onto an individual electron in Newton's law. This equation is solved numerically together with the Maxwell equations by a finite-difference time-domain approach. We use literature parameters [14] for the gold plasma frequency $\left(\omega_{\mathrm{pl}}=\sqrt{\mathrm{e}^{2} n_{\mathrm{i}} /\left(\varepsilon_{0} m\right)}=1.367 \times 10^{16} \mathrm{~s}^{-1}\right)$ and the 

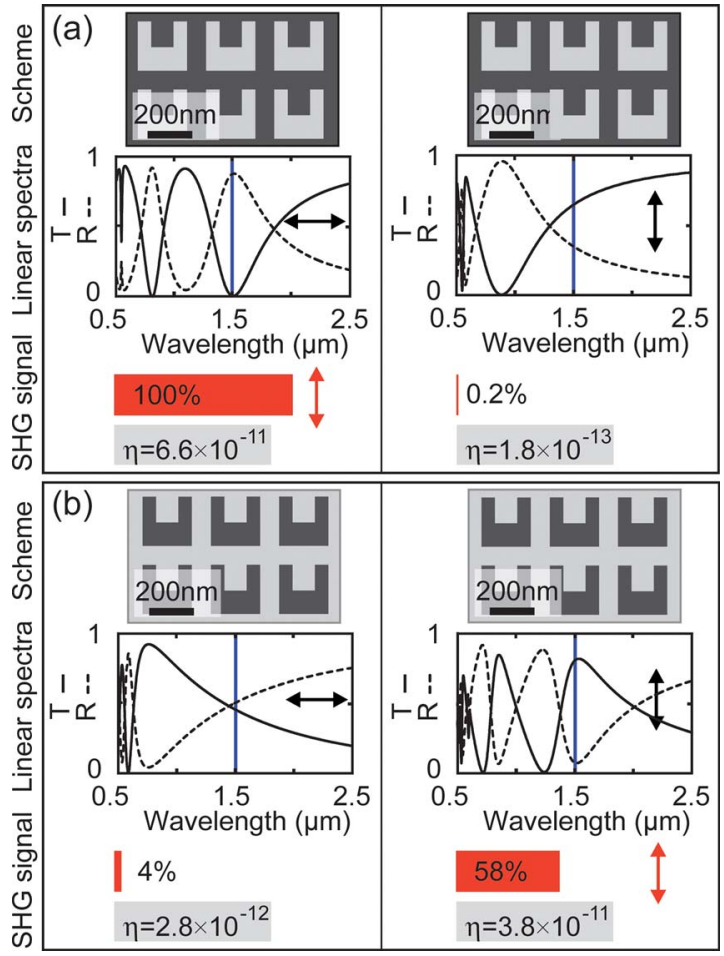

Fig. 2. (Color online) Theoretical results for (a) SRR and (b) CSRR that can directly be compared to the experimental results shown in Fig. 1. The absolute power conversion efficiencies $\eta$ are quoted at the bottom.

collision frequency $\left(\omega_{\text {coll }}=6.478 \times 10^{13} \mathrm{~s}^{-1}\right)$. The lateral geometrical SRR and CSRR parameters are indicated as insets in Fig. 2, and the gold thickness is $25 \mathrm{~nm}$. The structures are located on a glass substrate (permittivity $=2.25$ ), coated with a $5 \mathrm{~nm}$ thin ITO layer (permittivity=3.8). Importantly, we use a peak incident electric field of the $170 \mathrm{fs}$ Gaussian pulses centered around $1.5 \mu \mathrm{m}$ wavelength of $E_{0}=2 \times 10^{7} \mathrm{~V} / \mathrm{m}$ - a value that we have previously estimated for our experiments [7]. This altogether allows for calculating not only the relative but also the absolute SHG power conversion efficiencies $\eta$ without any adjustable parameters. Results are shown in Fig. 2, which can directly be compared with the experiment in Fig. 1. Obviously, the overall agreement with experiment is very good-especially if one considers the experimental uncertainties in determining $E_{0}$. By artificially switching on and off the different nonlinear source terms for SRR (CSRR), we find that the convective term accounts for about 68\% (81\%) of the SHG source current density $\vec{j}_{2 \omega}^{(2)}$. The corresponding source term based on the magnetic part of the Lorentz force [6] shows a similar qualitative behavior but accounts for only about $2 \%$ ( $2 \%$ for SRR).

In conclusion, we have presented a study of SHG from arrays of CSRR with SHG from arrays of SRR, both excited under normal incidence at their fundamental resonance. SRR and CSRR show comparable SHG, which is much larger than the SHG from any other structure that we have investigated so far. Our experimental results have been analyzed at the level of cold plasma electrodynamics theory, showing that the convective contribution dominates the secondorder nonlinear response. Regarding possible applications, the CSRRs are advantageous in that they reveal a transmittance maximum at the fundamental resonance rather than a minimum for the SRR. This larger transmittance potentially allows for stacking several layers along the propagation direction of light, thereby enhancing the SHG conversion efficiency.

We thank K. Busch, G. Shvets, and C. M. Soukoulis for discussions. The research for S. L. is further supported through a Hochschul-Nachwuchsgruppe (VHNG-232). The Arizona-Marburg work is supported by the DFG, the Air Force Office of Scientific Research, and a senior Humboldt award (J. V. M.). The project PHOME acknowledges the financial support of the Future and Emerging Technologies (FET) programme within the Seventh Framework Programme for Research of the European Commission, under FET-Open grant 213390. The project METAMAT is supported by the Bundesministerium für Bildung und Forschung (BMBF).

\section{References}

1. J. B. Pendry, A. J. Holden, D. J. Robbins, and W. J. Stewart, IEEE Trans. Microwave Theory Tech. 47, 2075 (1999).

2. V. M. Shalaev, Nat. Photonics 1, 41 (2007).

3. C. M. Soukoulis, S. Linden, and M. Wegener, Science 315, 47 (2007).

4. J. E. Sipe and R. W. Boyd, Phys. Rev. A 46, 1614 (1992).

5. V. M. Shalaev, Phys. Rep. 272, 61 (1996).

6. M. W. Klein, C. Enkrich, M. Wegener, and S. Linden, Science 313, 502 (2006).

7. M. W. Klein, M. Wegener, N. Feth, and S. Linden, Opt. Express 15, 5238 (2007).

8. M. W. Klein, M. Wegener, N. Feth, and S. Linden, Opt. Express 16, 8055 (2008).

9. S. Kujala, B. K. Canfield, M. Kauranen, Y. Svirko, and J. Turunen, Phys. Rev. Lett. 98, 167403 (2007).

10. T. Xu, X. Jiao, G. P. Zhang, and S. Blair, Opt. Express 15, 13894 (2007).

11. F. Falcone, T. Lopetegi, M. A. Laso, J. D. Baena, J. Bonache, M. Beruete, R. Marques, F. Martin, and M. Sorolla, Phys. Rev. Lett. 93, 197401 (2004).

12. T. Zentgraf, T. P. Meyrath, A. Seidel, S. Kaiser, H. Giessen, C. Rockstuhl, and F. Lederer, Phys. Rev. B 76, 033407 (2007).

13. Y. Zeng, W. Hoyer, J. Liu, S. W. Koch, and J. V. Moloney, "Classical theory of second-harmonic generation from magnetic metamaterials," arXiv:0807.3531v1 http://arxiv.org/abs/0807.3531v1 (2008).

14. C. Enkrich, M. Wegener, S. Linden, S. Burger, L. Zschiedrich, F. Schmidt, J. Zhou, Th. Koschny, and C. M. Soukoulis, Phys. Rev. Lett. 95, 203901 (2005).

15. J. Freidberg, Plasma Physics and Fusion Energy (Cambridge, 2007). 